\title{
El aprendizaje en la base de la pirámide: Restricciones, comparabilidad y política en países en vías de desarrollo
}

\author{
Learning at the bottom of the pyramid: \\ Constraints, comparability and policy in developing countries
}

\author{
Daniel A. Wagner y Nathan M. Castillo'
}

\section{Resumen}

Los objetivos para el desarrollo de las Naciones Unidas han asignado, de manera sostenida, una alta prioridad a la calidad educativa -y a la del aprendizaje-. Esto ha llevado a avances sustanciales en ayuda para el desarrollo internacional hacia la educación, y también a una mayor atención, a nivel mundial, a la importancia del aprendizaje de los niños. Sin embargo, tales metas son principalmente normativas: tienden a representar promedios entre naciones, brindando limitada atención a las variaciones dentro de dichos países. La presente investigación proporciona un análisis de las tensiones científicas en la comprensión del aprendizaje en poblaciones pobres y marginadas: aquellos en la base de la pirámide (BdP). Si bien agencias internacionales como UNESCO y OCDE a menudo invocan a estas poblaciones como el «objetivo» de sus inversiones y evaluaciones, continúan los debates importantes sobre la ciencia empírica involucrada tanto en la investigación como en las políticas. El presente análisis concluye que los objetivos para el desarrollo post-2015 de las Naciones Unidas tienen que tomar en cuenta la necesidad critica de enfocarse en el aprendizaje de los pobres a fin de abordar adecuadamente las inequidades sociales y económicas².

\section{Palabras clave}

Aprendizaje, países en vías de desarrollo, poblaciones pobres y marginalizadas, resultados de aprendizaje, educación comparativa, política educativa.

\section{Abstract}

United Nations development goals have consistently placed a high priority on the quality of education - and of learning. This has led to substantive increases in international development assistance to education, and also to broader attention, worldwide, to the importance of children's learning. Yet, such goals are mainly normative: they tend to be averages across nations, with relatively limited attention to variations within countries. This review provides an analysis of the scientific tensions in understanding learning among poor and marginalized populations: those at the bottom of the pyramid. While international agencies, such as UNESCO and OECD, often invoke these populations as the «target» of their investments and assessments, serious debates continue around the empirical science involved in both research and policy. The present analysis concludes that the UN post-2015 development goals must take into account the critical need to focus on learning among the poor in order to adequately address social and economic inequalities..

\section{Key words}

Learning, low-and middle-income countries, poor and marginalized populations, learning outcomes, constraints comparability, education policy

1 University of Pennsylvania, Daniel A. Wagner, wagner@literacy.upenn.edu; Nathan M. Castillo, ncast@gse.upenn.edu

2 Una versión anterior de este artículo apareció en Wagner y Castillo (2014). Agradecemos a Jazmín Mora por su ayuda con la traducción. 


\section{Introducción}

La Conferencia Mundial sobre Educación para Todos en Jomtien, Tailandia, fue un momento decisivo en la educación y desarrollo internacionales. Llevada a cabo en 1990, la Conferencia abarcó dos desafíos clave: aumentar significativamente el acceso a la educación para los niños de países pobres y promover la calidad de aprendizaje en la educación. Una década más tarde, en la conferencia Educación para Todos (EPT) de 2000 en Dakar, estos dos mismos desafíos fueron expandidos en una lista detallada de seis objetivos de educación en el Marco de Acción de EPT en Dakar. Los objetivos eran promover la atención y el cuidado de la primera infancia, hacer obligatoria la enseñanza primaria, abordar las necesidades de aprendizaje para todos, promover la alfabetización de adultos, reducir las disparidades de género y desarrollar mediciones de calidad de los resultados del aprendizaje (UNESCO 2003:28). Estos fueron reforzados de nuevo en los Objetivos de Desarrollo del Milenio (ODM) de las Naciones Unidas para el 2015, donde la educación primaria universal se convirtió en el segundo de ocho objetivos principales (Naciones Unidas, 2000). Estos esfuerzos globales llevaron a las agencias de desarrollo internacional a aumentar significativamente su asistencia a la educación y también llevó al público en general a prestar más atención al aprendizaje de los niños en una escala global.

Existe una gran y diversa base de investigación empírica en el área del aprendizaje humano. Sin embargo, gran parte de la información disponible se encuentra sustancialmente limitada por restricciones de diversos tipos. La restricción más prominente es la limitada capacidad de generalizar desde hallazgos en contextos de una población a contextos de una población distinta. Del mismo modo, los métodos de investigación pueden variar de gran manera entre conjuntos de estudios, lo que dificulta discernir si los resultados varían debido a los métodos o a otros factores. Estos son, por supuesto, problemas clásicos en las ciencias sociales.

En este artículo, analizamos las tensiones científicas en la comprensión del aprendizaje de las poblaciones pobres, aquellas que Prahalad (2006) llamó la base de la pirámide (BdP). Si bien las agencias internacionales a menudo sostienen que invierten en las poblaciones más necesitadas, continúan los serios debates sobre la ciencia empírica necesaria para implementar las políticas apropiadas, con consecuencias inevitables para la implementación efectiva en países de ingreso bajo y medio.

\section{Restricciones}

\section{El aprendizaje en dos aulas sudafricanas:}

La Escuela Primaria Shayandime, con edificios de paredes de adobe y techos de zinc, se encuentra ubicada en una pequeña aldea en la provincia septentrional de Limpopo, Sudáfrica. A tan solo unas docenas de millas de la frontera con Zimbabue, el área está salpicada con casas tradicionales llamadas rondaveles, una versión adaptada de la cabaña del estilo de África meridional. Los babuinos rondan por el recinto escolar libremente y son conocidos por deslizarse por el espacio entre las paredes de barro rojo y el techo corrugado y vandalizar las aulas por la noche. A pesar de la ocasional ventana rota, a la escuela no le faltan recursos. Es uno de los muchos establecimientos en la región que recibió una donación de antiguas computadoras de escritorio en las cuales los estudiantes de primaria superior pasan tiempo trabajando en actividades básicas de mecanografía. No obstante, los alumnos desfavorecidos con escasa competencia en inglés no tienen acceso a las computadoras, ya que las mismas carecen de programas en el idioma local, Venda. En el aula, los estudiantes pasan la mayoría de su tiempo copiando oraciones de la pizarra y rara vez participan de actividades estimulantes que apoyen la creatividad y las habilidades de pensamiento crítico. 
En contraste, a cuatro horas de distancia, en la capital provincial de Polokwane, se sitúa la Escuela Primaria Central. Con veredas de ladrillo alrededor del perímetro y con un laboratorio informático de vanguardia, la misma tiene monitores de pantalla plana y una pizarra inteligente con proyector. El laboratorio de informática, parecido al de la universidad local, fue adquirido en parte a través de las ganancias obtenidas al alquilar el salón de eventos de la escuela a la comunidad. No hay ventanas rotas, los profesores presentan planes de estudio estructurados y los padres conforman una parte integral de la cultura escolar. Teniendo en cuenta su atractivo ambiente de aprendizaje, los funcionarios provinciales exhiben orgullosamente esta escuela urbana a los planificadores educativos nacionales e internacionales en sus visitas. Muchos estudiantes tienen teléfonos móviles y dan la impresión de estar motivados a aprender y estar conectados con el futuro de Sudáfrica ${ }^{3}$.

Comparaciones de contextos rurales y urbanos en países en vías de desarrollo a menudo consisten en estos tipos de observaciones de características sociales y de infraestructura. La Evaluación Nacional Anual de Sudáfrica (ANA, por sus siglas en inglés), administrada al final de cada año escolar, mide el progreso en los logros de aprendizaje de los grados primero al sexto y al noveno (DBE, 2013). Esta tiende a confirmar la perspectiva subjetiva presentada más arriba. Las escuelas sudafricanas se clasifican según un índice de pobreza que está basado en la riqueza o pobreza relativa de la comunidad y se agrupan en quintiles. La Escuela Primaria rural Shayandime pertenece al quintil más bajo. Cuando allí se llevó a cabo la ANA, solo cinco estudiantes en el tercer grado obtuvieron puntajes por encima del promedio nacional, mientras que la gran mayoría obtuvo resultados en el 10\% inferior, creando así una distribución sesgada. En contraste, la Escuela Primaria Central se sitúa en el (tercer) quintil medio con resultados normalmente distribuidos; los mismos se encuentran en cierto modo por debajo de los promedios urbanos nacionales para las competencias en matemáticas y la alfabetización en la lengua materna (DBE, 2013).

El contraste en el desempeño de las pruebas ANA entre estas dos escuelas plantea dos preguntas relacionadas: ¿Por qué Shayandime tiene algunos estudiantes de alto rendimiento, con el resto de sus estudiantes agrupados alrededor del continuo de rendimiento más bajo? Y, dentro de la Escuela Primaria Central, ¿Por qué los puntajes se distribuyen de manera más normal, aunque por debajo del promedio nacional? Los estudios a menudo señalan la función que las influencias sociales y familiares tienen en predecir los resultados de aprendizaje, principalmente en términos de lo que llamamos poder, padres, y privilegio. Por ejemplo, varios autores han estudiado el impacto de la estratificación social en los resultados escolares en términos de tales factores (Benedict y Hoag, 2004; Buchmann y Hannum, 2001; Korinek y Punpuing, 2012; Lu yTreiman, 2011). En muchos casos, los estudiantes con la correcta combinación de estas influencias son los que tienden a sentarse más cerca del frente en aulas grandes y también tienen mayor concentración y motivación para aprender.

Estos hallazgos tienen implicaciones serias. Nuestro entendimiento de la ciencia psicológica del aprendizaje deriva principalmente de los datos de los países ricos de la OCDE, donde los resultados educativos, incluyendo aquellos de exámenes estandarizados son, gracias a su diseño, normalmente distribuidos. Sin embargo, la variación alrededor del mundo, y especialmente en contextos de la BdP como hemos observado en las zonas rurales de Sudáfrica, puede ser sesgada: unos cuantos puntajes altos y muchos estudiantes con puntajes bajos. En lugares donde el contraste es tan dramático, y donde el foco se encuentra en el extremo inferior de la curva normal, nos vemos obligados a reconsiderar las nociones de la

3 Nota del autor: los nombres de las escuelas han sido cambiados, y estos perfiles combinan detalles de varias escuelas 
normalidad estadística, y con ello, la idea que los contextos de la BdP son simplemente una extensión de la típica curva normal.

La idea de que el aprendizaje dentro y fuera de la escuela puede variar considerablemente entre culturas no es nueva (Cole, Gay, Glick y Sharp, 1971; Wagner, 1993, 2014). No obstante, el aumento continuo de la presión para globalizar la recopilación de datos sobre la educación ha llevado a los investigadores y a los responsables de las políticas públicas a ignorar, o minimizar tales diferencias (Benavot y Tanner, 2007). Creemos que la falta de consideración al aprendizaje en la base de la pirámide distorsiona la realidad y puede llevar a intervenciones desconsideradas a favor de los estudiantes pobres.

Más allá de Sudáfrica, abundan los ejemplos de estilos de aprendizaje distintivos en la literatura de investigación. Parte de este trabajo se originó hace décadas en los Estados Unidos con estudios pioneros sobre las diferencias individuales en el aprendizaje (Kagan, Moss y Sigel, 1963; Witkin, Moore, Goodenough y Cox, 1977). Hoy en día, los estudiantes de las escuelas pobres de países de ingreso bajo y medio a menudo se esfuerzan para entender el lenguaje de instrucción y el lenguaje de lectura. En tales situaciones, los profesores hacen énfasis en el aprendizaje mecánico y la memorización; aunque esta sigue siendo una estrategia de aprendizaje muy común en el mundo, es ridiculizada por los pedagogos modernos (occidentales) (Wagner, 1983). Otro ejemplo de estos contrastes es la manera en la cual el aprendizaje es construido en varias sociedades, como las que están fuertemente influenciadas por el Confucionismo (Li, 2003).

El aprendizaje es omnipresente y toma diferentes formas en la vida cotidiana. En la educación, el aprendizaje se mide con instrumentos que puedan estimar de forma fiable tanto los procesos como los resultados -o las evaluaciones de aprendizaje-. Si es necesario que una evaluación sea representativa de la población entera de un país, o válida en varios países en un marco comparativo, entonces probablemente costará más en términos de tiempo y recursos. Hasta ahora, los investigadores han controlado el tiempo y los recursos delimitando el rango de habilidades a ser evaluadas (la muestra de habilidades), y limitando la población a ser incluida (la muestra de la población). Es importante comprender estas dos formas de límites en términos de requerimientos técnicos y estadísticos, así como también los requerimientos y resultados de las políticas. Cada uno de estos temas plantea desafíos empíricos y estadísticos.

\section{Muestreos de habilidades y evaluaciones}

Es ampliamente aceptado que los humanos aprenden a través del muestreo de sus entornos, comenzando por el uso de sus sentidos incorporados desde el momento del nacimiento. Claramente, ningún niño, joven ni adulto podría sobrevivir asimilando la totalidad de la información disponible en el entorno. En otras palabras, los sistemas humanos están diseñados para discriminar información para así tomar muestras de la información que mejor les ayude a manejar los desafíos del aprendizaje (Kahneman, 2011). De hecho, los padres normalmente preparan a los niños pequeños a adaptarse, aprender y sobrevivir precisamente exponiéndolos a la gama de situaciones que encontrarán en sus vidas. Por supuesto, no todos estos entornos de aprendizaje pueden ser igualmente adaptables para el futuro del niño en ambientes educativos.

Cuando se trata de la investigación científica sobre el aprendizaje, nos va mejor a los seres humanos cuando tomamos muestras de nuestro ambiente informativo, ya sea de instituciones educativas, verbalmente o, cada vez más, a través de buscadores de internet como Google. Esta simple observación es muy relevante aquí: uno de los problemas más exasperantes que encuentran los investigadores al estudiar y evaluar el aprendizaje es como generalizar de una muestra de habilidades a otra. Por lo tanto, 
muestrear una colección finita de habilidades y comprender las situaciones contextuales en las cuales se utilizan son elementos claves de todas las evaluaciones de aprendizaje.

Al diseñar estrategias para la investigación y evaluación del aprendizaje, los investigadores toman decisiones altamente complejas: eligen variables contextuales y demográficas (por ejemplo edad del niño, año escolar, sexo, situación socioeconómica), y eligen las habilidades a ser evaluadas y el tipo de metodología de investigación a ser utilizada. Cada opción está ligada a varias suposiciones y concesiones, y las selecciones incluidas en el diseño final influenciarán la validez, fiabilidad y viabilidad práctica del enfoque elegido (Braun yKanjee, 2006; Wagner, 2010, 2011a). Además, la investigación debe ser diseñada de manera que pueda adaptarse a los cambios dinámicos a lo largo del tiempo. Y, a medida que las expectativas de alfabetización, aritmética y las capacidades de orden superior se adaptan a los cambios en los entornos sociales y económicos, los métodos de medición también deben ser adaptados para que se alineen con los objetivos educativos (Wagner, Murphy y de Korne, 2012).

\section{El aprendizaje y muestras de la población}

El muestreo de población también es importante. Por ejemplo, cerca del $5 \%$ de la población mundial reside en los Estados Unidos, pero alrededor del 95\% (Arnett, 2008) de las publicaciones científicas sobre el desarrollo psicológico son basadas en poblaciones que son OEIRD (occidentales, educadas, industrializadas, ricas y democráticas; Heinrich, Heine y Norenzayan, 2010), y viviendo principalmente en países OCDE. Además, de las investigaciones sobre desarrollo psicológico conducidas en los Estados Unidos, aproximadamente el $80 \%$ pertenece a grupos étnicos «mayoritarios» (de ascendencia europea), por más de que estos grupos representan solo el $50 \%$ de la población actual de los Estados Unidos (Arnett, 2008). Parece obvio que los investigadores deben abordar explícitamente las cuestiones de representatividad y validez externa, pero a menudo no lo hacen. Estas críticas también se aplican a la investigación internacional, ya que gran parte de la investigación disponible sobre el aprendizaje está importantemente restringida por bases de datos científicos y estudios de investigación extraídos de muestras de poblaciones que viven en países más desarrollados. Afortunadamente, esta tendencia está empezando a cambiar (Wagner, 2014).

En las evaluaciones educativas internacionales a gran escala (LSEAs por sus siglas en inglés) partes claves de las poblaciones de la BdP pueden verse excluidas o sub-representadas en muestras que se consideran nacionales (Engel yFeuer, 2014; Wagner, 2011b). El género ha sido una de las principales razones por la cual los niños no asisten a la escuela en los países en vías desarrollo, aunque en las últimas décadas se han registrado avances significativos. Aun así, en los países más pobres hay menos niñas que niños presentes en escuelas en los dos momentos donde normalmente los datos están recogidos: el comienzo de la escuela primaria y la secundaria. La exclusión sistemática de niñas en países pobres tiende a resultar en una menor cantidad de niñas adolescentes que asisten a la escuela; aquellas que si asisten a menudo obtienen resultados más bajos en evaluaciones nacionales en comparación con los varones. Por ejemplo, según los resultados del sexto grado de la evaluación regional del Consorcio Africano Meridional y Oriental para el Seguimiento de la Calidad Educativa (SACMEQ por sus siglas en español), llevada a cabo en el 2007, Saito (2011) encontró que, a base de un promedio de 15 países africanos, los niños generalmente superaban a las niñas en matemáticas, mientras que las niñas superaban a los niños en la alfabetización. Sin embargo, las diferencias nacionales en las disparidades de género varían ampliamente tanto en alfabetización como en matemáticas. Tendencias semejantes surgen en las evaluaciones nacionales que sobre-muestrean las zonas urbanas de fácil acceso en países 
pobres. Además, en algunos países en vías de desarrollo, la dificultad de rastrear a niños nómadas puede ser onerosa y costosa como para que las autoridades educativas puedan incluirlos en las escuelas (UNESCO, 2010).

Otro problema que existe en casi todos los países es la variación de idioma entre los grupos étnicos. Muchos de estos grupos, a veces denominados minorías etnolingüísticas, están bien integrados en una «mezcla» nacional, como en Suiza; pero en otras situaciones estas variaciones pueden contribuir a conflictos civiles. En Latinoamérica, con más de 500 idiomas indígenas, es una región donde la educación intercultural bilingüe se está expandiendo para promover el cambio social; hasta la fecha, 12 gobiernos han institucionalizado la pedagogía multilingüe (Cortina, 2014). A menudo, las fuerzas sociales y políticas intentan ayudar a resolver las diferencias, generalmente incluyendo decisiones políticas que resultan en una jerarquía de idiomas aceptables para el uso en escuelas y estructuras de gobierno. En tales situaciones, ya sea en países de la OCDE o en países en vías de desarrollo, no es raro que los niños que hablan idiomas minoritarios sean excluidos de la investigación y evaluaciones de aprendizaje.

Este proceso de exclusión también ocurre en regiones donde los conflictos civiles o las crisis económicas provocan una migración transfronteriza sustancial, donde los grupos inmigrantes (y sus hijos) son tratados como transitorios, y donde los niños reciben poca o ninguna educación (Pigozzi, Carrol, Hayden y Ndaruhutse, 2014). El Informe de Monitoreo Global del 2010 describe como la marginación puede amenazar el logro educativo, ya que estos niños enfrentan muchos desafíos. En general los alumnos más marginados del mundo se enfrentan a «desigualdades, estigmatización y discriminación vinculadas a la riqueza, el género, la etnicidad, el idioma, la ubicación y la discapacidad» (UNESCO, 2010:5). La magnitud en que estos grupos están, o no están, incluidos en muestras de la población tiene implicaciones graves a medida que los investigadores desarrollan normas para los resultados del aprendizaje. La mayoría de los que forman parte de la población de interés pueden tratar a los «otros» como un grupo inferior que «no puede aprender». Irónicamente, en Sudáfrica, donde los pobres están en la mayoría, son los estudiantes rurales y pobres los que se sienten más marginados y desamparados (Babson, 2010). En resumen, tanto las muestras de habilidades como de población varían, así como también los procesos de aprendizaje que los individuos implementan y los contextos en los cuales estos tienen lugar.

Finalmente, debemos considerar agentes interesados que realizan el muestreo. Ya sea se trate de aquellos que diseñan las políticas públicas, psicómetras o profesores locales, todos estos llegan a la tarea de muestrear habilidades y poblaciones desde sus propios puntos de vista y experiencias. Las decisiones sobre que habilidades muestrear, entre que poblaciones, y en que idiomas y contextos, también contribuyen a un posible sesgo o a un ya complejo juego de asuntos de muestreo. Para hacer frente a estos sesgos, los investigadores pueden usar un rango de métodos incluyendo muestreo a medida y diseños de submuestras; las muestras de concordancia, el sobre-muestreo de poblaciones marginadas y diseños de métodos mixtos. Las consecuencias de estas varias restricciones pueden tener un impacto importante en la política y practica pedagógica, y en la gobernanza mundial de la educación (Meyer y Benavot, 2013).

\section{Credibilidad Metodológica}

La investigación que puede convertirse en política depende de su credibilidad, lo que significa que especialistas debidamente capacitados deben llegar a un consenso sobre los méritos de un conjunto particular objetivo de resultados, incluso si no están de acuerdo con la interpretación de dichos resultados. 
Las dos dimensiones más citadas de la credibilidad en la investigación del aprendizaje son la validez y la fiabilidad.

La validez de cualquier herramienta de medición de aprendizaje se puede determinar de varias maneras. En primer lugar, la validez interna está determinada por el grado en que los hallazgos pueden relacionarse de manera creíble con la lógica conceptual de la intervención minimizando el error sistemático, o sesgo. Por ejemplo, ¿Se relacionan realmente las preguntas en una prueba de opción múltiple con la capacidad del niño de leer o con la capacidad de recordar lo que él o ella ha leído antes? La validez puede variar significativamente según el contexto y la población, ya que una prueba que puede ser válida en Londres puede tener poca validez en Lahore. Del mismo modo, una prueba efectiva para un grupo lingüístico de hablantes de lengua materna puede ser inapropiada para niños que hablan ese mismo idioma como segunda lengua. Esta segunda forma de validez se denomina apropiadamente validez externa: se refiere a la replicabilidad de los hallazgos a través de distintos contextos. Si las estadísticas continúan acumulándose sin tener en cuenta el contexto local, las evaluaciones pueden malinterpretar a los alumnos en contextos de la BdP.

Se ha planteado un tercer tipo de preocupación por la validez con respecto a los países en vías de desarrollo: ¿hasta qué punto son válidas las opciones de los ítems de prueba y cuán apropiado es su contenido cuando son aplicadas a las culturas y a los sistemas escolares locales? Si bien muchas investigaciones de aprendizaje toman la forma de pruebas cuantitativas, los métodos cualitativos y etnográficos también pueden contribuir, particularmente con respecto a las variaciones culturales en el proceso de aprendizaje en contextos diversos.

A menudo, la fiabilidad se mide de dos maneras cuantitativas. En general, la confiabilidad es el grado en que los resultados de un individuo en una prueba están constantemente relacionados en tiempos adicionales en los que el individuo toma la misma prueba (o equivalente). Generalmente, la alta fiabilidad significa que el orden de rango de individuos que toman una prueba sería muy similar en una segunda ocasión que la tomen. Una segunda manera más fácil de medir la fiabilidad es mirar la función interna de los ítems de la prueba: ¿Tienen los ítems en cada parte de la evaluación una fuerte asociación entre sí? Esto es conocido como la confiabilidad entre-ítem, medida por la estadística alfa de Cronbach. Por supuesto, la fiabilidad implica poco sobre la validez del instrumento: el consenso de los investigadores de que el instrumento es relevante a los resultados educativos.

Desde el punto de vista cualitativo, la fiabilidad se alcanzaría cuando los etnógrafos con sensibilidad al contexto, por ejemplo, se pusieran de acuerdo en un conjunto de observaciones de procesos de aprendizaje que hayan recolectado de manera independiente en un contexto particular. Este es un ejemplo de «etnografía de equipo» que se está utilizando cada vez más en la investigación educativa en los Estados Unidos y Europa (Bartlett y Garcia, 2011; Blackledge y Creese, 2010). Además, el uso de experimentos controlados aleatorios (ECA) es visto como una manera importante de aumentar la credibilidad de los resultados de la investigación, comparando intervenciones con otros grupos de control. Evaluaciones hechas por Kremer y Holla (2009), Banerjee y Duflo (2011) y Bruns, Filmer y Patrinos (2011) apoyan el uso de ECA para mejorar la credibilidad de las investigaciones en el desarrollo internacional, mientras que otros (por ejemplo, Castillo y Wagner, 2014) sugieren algunas limitaciones graves en el uso de ECA para el diseño de la política educativa.

La diversidad de los resultados de aprendizaje se resume a menudo en términos de un rango promedio o normal que puede ser aplicado a lo largo de las dimensiones predecibles de una curva campaniforme 
(Gurn, 2010). Como muchos han indicado, la conclusión que el comportamiento humano cae a lo largo de la curva normal, con la mayoría de las observaciones concentradas alrededor de un promedio discernible, simplifica excesivamente el rango y la diversidad de las experiencias humanas (Dudley-Marling y Gurn, 2010). La sociedad y la cultura influencian casi todos los aspectos de la condición humana, desde la inteligencia hasta la altura y el peso, de muchas maneras no aleatorias. En el ámbito del aprendizaje y desarrollo internacional, la sobre dependencia en interpretar los hallazgos a través de una perspectiva de la distribución normal contiene sesgos inherentes.

¿Por qué nos importa? Es potencialmente engañoso basar afirmaciones sobre el aprendizaje humano, y hacer predicciones al respecto, que estén basadas en una suposición de distribuciones normales. Hemos sostenido que, para los estudiantes en la BdP, el aprender ciencia puede ser sustancialmente diferente de aquellas poblaciones más favorecidas. Una estrategia útil podría ser enfocarse en lo que los estudiantes en la BdP contribuyen al proceso del aprendizaje en lugar de enfocarse en lo que les falta. Por ejemplo, Harper (2012) enmarca la investigación anti-déficit en contextos de educación de los Estados Unidos, además Moll, Amanti, Neff y González (1992) describen una orientación que examina los «fondos del conocimiento» o activos. Cada uno de estos enfoques respalda la noción de que es necesario centrarse más en qué y cómo se lleva a cabo el aprendizaje en la base de la pirámide.

\section{Comparabilidad de resultados de aprendizajes entre contextos}

La comparabilidad es central en las bases de datos globales de educación, tales como la colección de datos a gran escala realizada por el Instituto de Estadística de UNESCO (UIS) y la OCDE. Sin embargo, si la meta principal es la comparabilidad, se puede prestar menos atención a la validez local y cultural de las definiciones y clasificaciones del aprendizaje. Además, los datos pueden volverse menos significativos y posiblemente menos aplicables a nivel local. Esta es una tensión natural y esencial entre los enfoques de la medición universalistas etic y los dependientes del contexto emic, y es particularmente relevante para el estudio de las poblaciones de la BdP. En un ejemplo conocido, los enfoques emic son aquellos que están conscientemente centrados en relevancia cultural y local, tales como palabras o descripciones locales para una persona «inteligente». Los enfoques etic son aquellos que pueden definir «inteligencia» como un concepto universal y tratar de medir a individuos entre culturas con ese único concepto o definición.

¿Pueden la comparabilidad y la sensibilidad al contexto estar balanceadas apropiadamente en la investigación del aprendizaje? ¿Deberían ser examinados los países con resultados por debajo del promedio usando las mismas escalas que países con resultados mucho más altos que el promedio? Si algunos países, o grupos de estudiantes, se encuentran en el «piso» de la escala, algunos dirían que la solución es bajar la escala a un nivel menor de dificultad. Otros podrían decir que la escala misma es defectuosa y que hay diferentes habilidades que podrían evaluarse mejor, especialmente si las variaciones son evidentemente causadas por variables culturales, étnicas, lingüísticas y variables relacionadas que llevan a uno a cuestionar la prueba tanto o más que el mismo grupo que está siendo examinado. Aun así, algunos dicen que tener diferentes escalas para diferentes grupos o naciones es un compromiso inaceptable de los puntos de referencias que buscan los formuladores de políticas internacionales, como el grupo operativo de aprendizaje métrico (Learning Metrics Task Force, Brookings Institution, 2013) o la Iniciativa Mundial para la Educación ante Todo de las Naciones Unidas (GEFI por sus siglas en inglés, 2014). Si el objetivo más importante es mejorar el aprendizaje en la BdP, ¿cuán creíbles son los hallazgos en la cola de la distribución de las evaluaciones internacionales (o incluso nacionales)? 
En la medida en que se puede lograr la comparabilidad (y ninguna evaluación de aprendizaje afirma tener una comparabilidad perfecta), los resultados permiten a los responsables de la formulación de políticas considerar su propia situación nacional o regional en relación con otras. Esto parece tener más mérito cuando las opciones a ser tomadas se aplican a situaciones proximales, más que a las distales. Por ejemplo, consideren un país de África que ha adoptado un programa particular de educación bilingüe que parece funcionar bien en la escuela primaria. Si el ministro de educación de un país vecino cree que el caso es lo suficientemente parecido a su contexto nacional, entonces tiene sentido comparar los resultados en, por ejemplo, las pruebas de alfabetización en la escuela primaria. Una comparación distal podría ser observar que un cierto programa bilingüe de educación en Canadá parece ser eficaz, pero dudar de la posibilidad de aplicarlo en un contexto muy diferente en África. Pero la proximidad no siempre es la característica más pertinente; por ejemplo, la competición entre los resultados educativos y los sistemas económicos de los Estados Unidos y Japón han sido el motivo de discusiones y debates prominentes durante muchos años (Stevenson and Stigler, 1982). En un ejemplo más reciente, los altos funcionarios de Botsuana estaban interesados en saber cómo Singapur llegó a conseguir los mejores puntajes en matemáticas en varios LSEAs (Gilmore, 2005; ver también Sjoberg, 2007).

La cuestión clave aquí es el grado en que es necesario tener comparabilidad completa en los resultados del aprendizaje, con todos los individuos y grupos en las mismas escalas de evaluación. O si se elige no «forzan» las concesiones necesarias para una sola escala unificada, ¿cuáles son las ganancias y las pérdidas en términos de comparabilidad? ¿Pueden mantenerse los objetivos internacionales y las estadísticas proporcionales como estables y fiables si se eligen enfoques localizados sobre la comparabilidad internacional? Las respuestas a estas preguntas han llevado a situaciones donde algunos países en vías de desarrollo pueden estar tentados a participar en evaluaciones internacionales de aprendizaje, pero dudan en hacerlo porque sus resultados pueden resultar ser muy bajos en comparación. O bien, pueden concluir que el costo de participar no aumenta suficiente valor a la toma de decisiones a nivel nacional (Greaney y Kellaghan, 1996). Otros pueden participar porque no quieren ser vistos como que tienen índices de referencia inferiores a aquellos usados en países de la OCDE; para ver la reciente discusión de algunos de estos asuntos, ver OCDE (2014) y Bloem (2013).

Finalmente, la investigación internacional sobre el aprendizaje requiere alguna forma de comparabilidad, pero tal vez de formas más variadas de las que se consideran generalmente hoy en día. Por ejemplo, las evaluaciones internacionales y regionales pretenden específicamente a la comparabilidad entre países, mientras que las evaluaciones híbridas (Wagner, 2011b) están más enfocadas en contextos locales y en una mayor validez. Estas últimas intentan combinar aspectos de evaluaciones a gran escala con las de escala local y pueden considerarse más pequeñas, rápidas y baratas. Una de las primeras evaluaciones híbridas fue el proyecto de evaluación de la alfabetización de UNESCO (ILI, 2002); versiones posteriores pueden verse en las evaluaciones de alfabetización temprana que han crecido en popularidad (Gove y Wetterberg, 2011). Las evaluaciones híbridas ofrecen una validez localizada que las evaluaciones a gran escala no ofrecen y pueden brindar resultados más específicos para mejorar el aprendizaje y las intervenciones entre las poblaciones pobres y marginadas. La clase de comparabilidad de mayor importancia depende de las metas políticas de interés, así como también de las consideraciones del calendario y recursos. 


\section{5. ¿Qué funciones desempeñan las partes interesadas?}

Muchos funcionarios interesados -incluyendo los formuladores de políticas, los ministros de educación, los líderes comunitarios en las aldeas rurales, los profesores, los padres y los especialistas en educacióndeberían de ser responsables por el contenido y el proceso de aprendizaje de los niños. Los editores de revistas académicas y las universidades pueden desempeñar un papel al requerir que los investigadores ofrezcan más explicaciones intencionales de la representación y las implicaciones inherentes de las muestras que incluyen en sus estudios publicados. Sin embargo, incluso hoy, los especialistas en educación y los estadísticos en la mayoría de los países han sido los «guardianes» de los procesos de aprendizaje y su importancia para el éxito escolar y económico. Una de las principales razones de este acceso restringido al conocimiento sobre el aprendizaje es la complejidad de la ciencia empírica del aprendizaje, como se indicada anteriormente.

Una segunda razón es la falta de conocimiento -y a veces creencias erróneas- entre padres e hijos sobre cuán importante es el aprendizaje y la educación para sus oportunidades de vida. Mucha evidencia, de muchas sociedades, sugiere que las personas en comunidades pobres subestiman el valor del aprendizaje y la educación escolar; por ejemplo, Stevenson y Stigler (1982) compararon las creencias de los padres en Estados Unidos, China y Japón. Hoy en día, es más importante que nunca involucrar a múltiples actores en la toma de decisiones educativas. En muchos países, el público se ha interesado más por el aprendizaje de los niños y los logros escolares en perspectiva comparativa, probablemente debido a la creciente globalización, a la influencia de las agencias internacionales, a los esfuerzos de las organizaciones no gubernamentales (ONGs), al mayor activismo comunitario y al interés de los padres. Algunos estudios de campo han involucrado un fuerte compromiso comunitario que ha llevado a los gobiernos a incorporar los hallazgos para el cambio de políticas; véase Bhattacharjea, Wadhwa, y Banerji (2011) en India, y Piper y Korda (2010) en Liberia.

Este tipo de intercambio de información a varios niveles es otra manera de vincular la ciencia con la responsabilidad y las expectativas. ¿A quién debe preocuparle si un niño, maestro, escuela, distrito o nación no se está desempeñando en un nivel dado de aprendizaje? De hecho, ¿cómo siquiera se construyen tales expectativas? ¿Cuáles expectativas se deben de tomar en cuenta? El conocimiento sobre la importancia del aprendizaje -y como este puede lograrse en contextos formales e informales y de manera estructurada e informal- tiene la posibilidad de abrir nuevos caminos en la investigación, el desarrollo de políticas, la participación comunitaria y familiar, y la apropiación local. No hay lugar donde esto sea más evidente que en la base de la pirámide, donde recientemente los padres y comunidades están tomando conciencia del papel que el aprendizaje puede desempeñar en la vida de sus hijos.

\section{Conclusiones Políticas}

En principio, las investigaciones sobre cómo mejorar el aprendizaje en países de bajos recursos y en comunidades pobres y marginadas - poblaciones de la BdP- no son más difíciles de llevar a cabo que las investigaciones semejantes en países más desarrollados. Sin embargo, teniendo en cuenta donde la mayoría de los recursos científicos (humanos y fiscales) se encuentran (en su mayoría en países de la OCDE), puede ser mucho menos conveniente para aquellos con la formación avanzada que se necesita para realizar el trabajo. Ese hecho, entre otros, es por qué aún queda mucho por saber sobre el aprendizaje en contextos de la BdP. 
La manera en la cual el aprendizaje es estudiado en los países en vías de desarrollo, y específicamente en poblaciones de la BdP, podría tener una gran importancia científica para los investigadores y los funcionarios de la educación. A medida que avanzamos desde Jomtien, Dakar, y los ODM de las Naciones Unidas hacia los objetivos de desarrollo posteriores al 2015, queda claro que las inequidades sociales y económicas persistirán a menos que mantengamos un enfoque serio en el aprendizaje entre los pobres. En su libro seminal sobre nuevos enfoques para llegar a los mercados de consumo de la BdP, C.K. Prahalad (2006) desafió a las corporaciones a adoptar una nueva filosofía de prestación de servicios para las poblaciones históricamente ignoradas. Al transformar la manera en que el aprendizaje es entendido en contexto de la base de la pirámide, podemos empezar a entender cómo mejorar la promoción de políticas para aumentar la calidad educativa e incrementar los resultados del aprendizaje entre aquellos que son más difíciles de acceder.

\section{Referencias bibliográficas}

Arnett, Jeffrey Jensen (2008): “The neglected 95\%: Why American psychology needs to become less American”. American Psychologist, 63 (7), 602-614.

Babson, A. (2010). The place of English in expanding repertoires of linguistic code, identification and aspiration among recent high school graduates in Limpopo Province, South Africa. Ph.D. dissertation: University of Michigan, Ann Arbor.

Banerjee, Abhijit V. y Duflo, Esther (2011). Poor economics: A radical retbinking of the way to fight poverty. New York: Public Affairs.

Bartlett, Lesley y Garcia, Ofelia (2011). Additive schooling in subtractive times: Bilingual education and Dominican immigrant youth in the Heights. Nashville: Vanderbilt University Press.

Benavot, A. y Tanner, E. (2007). The growth of national learning assessments in the world, 1995-2006. Background paper prepared for EFA Global Monitoring Report 2008. Paris: UNESCO.

Benedict, Mary Ellen y Hoag, John (2011): "Seating location in large lectures: Are seating preferences or location related to course performance?” The Journal of Economic Education, 35 (3), 215-239.

Bhattacharjea, Suman; Wadhwa, Wilima y Banerji, Rukmini (2011). Inside primary schools: A study of teaching and learning in rural India. New Delhi: ASER. http://img.asercentre.org/docs/Publications/ Inside_Primary_School/Report/tl_study_print_ready_version_oct_7_2011.pdf

Blackledge, Adrian y Creese, Angela (2010). Multilingualism: A critical perspective. London: Continuum.

Bloem, S. (2013): "PISA in Low and Middle Income Countries". OECD Education Working Papers, 93.

Braun, Henry y Kanjee, Anil (2006): “Using assessment to improve education in developing nations” en Joel E. Cohen, David E. Bloom, \& Martin B. Malin (eds.): Educating all children: A global agenda. Cambridge, MA: MIT Press.

Brookings Institution (2013). Toward universal learning: Recommendations from the Learning Metrics Task Force. Washington, DC: Brookings Institution Center for Universal Education. http:// www.brookings.edu/ /media/Research/Files/Reports/2013/09/learning\%20metrics\%20task\%20 force $\% 20$ universal $\% 20$ learning/LTMF\%20RecommendationsReportfinalweb.pdf 
Bruns, Barbara; Filmer, Deon y Patrinos, Harry Anthony (2011). Making schools work: New evidence on accountability reforms. Washington: World Bank.

Buchmann, Claudia y Hannum, Emily (2001): "Education and stratification in developing countries: A review of theories and research". Annual Review of Sociology, 27, 77-102.

Castillo, Nathan M. y Wagner, Daniel A. (2014): “Gold standard? The use of randomized controlled trials for international educational policy". Comparative Education Review, 58 (1), 166-173.

Cole, M.; Gay, J.; Glick, J. y Sharp, D. (1971). The cultural context of learning and thinking. New York: Basic Books.

Cortina, Regina (ed.) (2014). The education of indigenous citizens in Latin America. Clevedon: Multilingual Matters.

DBE [Department of Basic Education], South Africa (2013). Report on the Annual National Assessment of 2013: Grades 1 to 6 \& 9. Pretoria: DBE. http://www.education.gov.za/LinkClick.aspx?fileticket= Aiw7HW8ccic\%3D\&tabid=36

Dudley-Marling, Curt y Gurn, Alex (eds.) (2010). The myth of the normal curve. New York: Peter Lang.

Engel, L. C. \& Feuer, M. J. (2014): "Five myths about international large-scale assessments". Quality Assurance in Education, 22, 18-21.

GEFI [Global Education First Initiative] (2014). Global Education First Initiative: An initiative of the United Nations Secretary-General. http:/ /www.globaleducationfirst.org/files/GEFI_Brochure_ENG.pdf

Gilmore, A. (2005). The impact of PIRLS (2001) and TIMSS (2003) in low - and middle-income countries: An evaluation of the value of World Bank support for international surveys of reading literacy (PIRLS) and mathematics and science (TIMSS). New Zealand: IEA.

Gove, Amber y Wetterberg, Anna (2011). The early grade reading assessment: Applications and interventions to improve basic literacy. Research Triangle Park, NC: RTI.

Greaney, Vincent y Kellaghan, Thomas (1996). Monitoring the learning outcomes of education systems. Washington: World Bank.

Gurn, Alex (2010): “Conclusion: Re/visioning the ideological imagination in (special) education” en Curt Dudley-Marling \& Alex Gurn (eds.): The myth of the normal curve. New York: Peter Lang.

Harper, Shaun R. (2012). Black male student success in higher education: A report from the National Black Male College Achievement Study. Philadelphia: University of Pennsylvania, Center for the Study of Race and Equity in Education.

Heinrich, Joseph, Heine, Steven y Norenzayan, Ara (2010): “The weirdest people in the world?” Behavioral Brain Science, 33 (2-3), 85-135.

Kagan, J., Moss, H.A. y Sigel, I.E. (1963): "Psychological significance of styles of conceptualization". Monographs of the Society for Research in Child Development, 28 (4), 73-112.

Kahneman, Daniel (2011). Thinking, fast and slow. New York: Macmillan. 
ILI [International Literacy Institute] (2002). Towards guidelines for the improvement of literacy assessment in developing countries: Conceptual dimensions based on the LAP Project. Philadelphia: ILI/UNESCO.

Korinek, Kim \& Punpuing, Sureeporn (2012): “The effect of household and community on school attrition: An analysis of Thai youth". Comparative Education Review, 56 (3), 474-510.

Kremer, Michael y Holla, Alaka (2009): "Improving education in the developing world: What have we learned from randomized evaluations?” Annual Review of Economics, 1, 513-542.

Li, Jin (2003): “U.S. and Chinese cultural beliefs about learning”. Journal of Educational Psychology, 95, 258-267.

Lu, Yao y Treiman, Donald J. (2011): "Migration, remittances and educational stratification among Blacks in Apartheid and Post-Apartheid South Africa". Social Forces, 89 (4), 1119-1143.

Meyer, Heinz-Dieter y Benavot, Aaron (eds) (2013). PIS A, power and policy: The emergence of global educational governance. Oxford: Symposium Books.

Moll, Luis C.; Amanti, Cathy; Neff, Deborah y Gonzalez, Norma (1992): "Funds of knowledge for teaching: Using a qualitative approach to connect homes and classrooms". Theory into Practice, 31 (2), 132-141.

OECD (2014). PISA for development. Paris: OECD. http://www.oecd.org/pisa/aboutpisa/pisafordevelopment.htm

Pigozzi, Mary Joy; Carrol, Bidemi, Hayden, Jacqueline y Ndaruhutse, Susy (2014): "Fragile and conflictaffected situations" en Daniel A. Wagner (ed.): Learning and education in developing countries: Research and policy for the post-2015 UN development goals. New York: Palgrave Macmillan.

Piper, Benjamin y Korda, Medina (2010). Early Grade Reading Assessment (EGRA) Plus: Liberia. Program evaluation report: Prepared for USAID/Liberia. Research Triangle Park, NC: RTI International.

Prahalad, C.K. (2006). The fortune at the bottom of the pyramid: Eradicating poverty through profits. Upper Saddle River: Wharton School Publishing.

Saito, M. (2011). Gender equality in education: Looking beyond parity. Paris: UNESCO-IIEP. http:// www.sacmeq.org/sites/default/files/sacmeq/publications/7_1_1_5_mioko_saito_eng_version_2012_02_15.pdf

Sjøberg, Svein (2007). "PISA and 'real life challenges': Mission impossible?" en S. T. Hopmann, G. Brinek \& M. Retzl (eds.): PIS A according to PIS A: Does PIS A keep what it promises? Vienna: LIT Verlag. http:/ / folk.uio.no/sveinsj/Sjoberg-PISA-book-2007.pdf.

Stevenson, Harold W. y Stigler, James W. (1982). The learning gap: Why our schools are failing and what we can learn from Japanese and Chinese education. New York: Summit.

UNESCO (2003). Education for All Global Monitoring Report: Gender and Education for All - The leap to equality. Paris: UNESCO.

UNESCO (2010). Education for All Global Monitoring Report: Reaching the marginalized. Paris: UNESCO.

United Nations (2000). United Nations millennium declaration. Resolution adopted by the General Assembly. United Nations A/RES/55/2. http://www.un.org/millennium/declaration/ares552e.htm 
Wagner, Daniel A. (1983): Rediscovering 'rote': Some Cognitive and Pedagogical Preliminaries en S. Irvine y J. W. Berry (eds.): Human assessment and cultural factors. New York: Plenum.

Wagner, Daniel A. (1993). Literacy, culture and development: Becoming literate in Morocco. New York: Cambridge University Press.

Wagner, Daniel A. (2010): "Quality of education, comparability, and assessment choice in developing countries". COMPARE: A Journal of Comparative and International Education, 40 (6), 741-760.

Wagner, Daniel A. (2011a): "What happened to literacy? Historical and conceptual perspectives on literacy in UNESCO”. International Journal of Educational Development, 31, 319-23.

Wagner, Daniel A. (2011b). Smaller, quicker, cheaper: Improving learning assessments in developing countries. Paris and Washington: UNESCO IIEP and EFA Fast Track Initiative of Global Partnership for Education.

Wagner, Daniel A. (ed.). (2014). Learning and education in developing countries: Research and policy for the post-2015 UN Development Goals. New York: Palgrave Macmillan.

Wagner, Daniel A.; Castillo, Nathan M. (2014): "Learning at the bottom of the pyramid: Constraints, comparability and policy in developing countries". Prospects: Quarterly Review of Comparative Education, 44, 627-638.

Wagner, Daniel A.; Murphy, Katie M. y de Korne, Haley (2012). Learning first: A Research Agenda for Improving Learning in Low-Income Countries. Washington: Brookings Institution.

Witkin, H. A.; Moore, C. A.; Goodenough, D. R. y Cox, P. W. (1977): "Field-Dependent and Field-Independent Cognitive Styles and their Educational Implications”. Review of Educational Research, 47, 1-64.

\section{Notas biográficas}

Daniel A. Wagner es UNESCO Chair, profesor de educación, y director del Instituto Internacional de Alfabetismo y el Programa Internacional de Desarrollo Educativo en la University of Pennsylvania (Estados Unidos). El Dr. Wagner tiene una extensa experiencia en temas educativos nacionales e internacionales y ha servido como asesor a UNESCO, UNICEF, el Banco Mundial, USAID, DFIC, y otras agencias en cuestiones de desarrollo internacional. El Dr. Wagner es Fellow de la Asociación Americana de Psicología, la Asociación Americana de Antropología y la Asociación Americana de Investigación Educativa.

Nathan M. Castillo es Doctor en Educación por la University of Pennsylvania (Estados Unidos) y Máster en Política Educativa Internacional de la University of Harvard. Actualmente es investigador asociado en el Instituto Internacional de Alfabetismo de la University of Pennsylvania. Anteriormente, ha trabajado en el Centro para el Desarrollo de la Educación en Washington, DC. Del 2006 al 2008, fue coordinador municipal para la salud escolar mientras servía en el Cuerpo de Paz de los Estados Unidos en Guatemala. 